$\xi=-1$

\title{
Spray Characteristics and Internal Flow Structures of Swirl Effervescent Atomizer
}

\author{
Z. A. Ghaffar, S. Kasolang*, Ahmad H. A. Hamid \\ Faculty of Mechanical Engineering, Universiti Teknologi MARA Selangor, 40450 Shah Alam, Malaysia \\ *Corresponding author E-mail: salmiahk@salam.uitm.edu.my
}

\begin{abstract}
Spray characteristics and internal flow structures of an atomizer are important parameters in indicating their performances. Both parameters are affected by the operating conditions and atomizer geometries. This study investigates the effect of operating parame ters and atomizer geometries on spray angle, breakup length and gas core diameter of swirl effervescent atomizer. This work is conducted specifically to find the most critical parameters that will produce the required spray characteristics and internal flow structure. The atomizer performance tests were carried out using water as the working fluid and nitrogen gas as the atomizing agent. High-speed shadowgraph technique was utilized to record the videos of the spray and internal flow structures. The video recordings were converted to image sequences and analyzed using image processing software. Geometrical parameters were found to be more dominant for characterizing the resultant sprays, but operating conditions was more critical in affecting the internal flow structures. The widest spray angle $\left(\sim 25^{\circ}\right)$ and shortest breakup length $(\sim 10 \mathrm{~mm})$ were observed at the high level of swirl-generating vane angle and discharge orifice diameter. Gas core diameter expanded up to $1.13 \mathrm{~mm}$ at the high level of GLR.
\end{abstract}

Keywords: Swirl Effervescent Atomizer; Spray Characteristics; Internal Flow Structures; Shadowgraph.

\section{Introduction}

Spray characterization is the process of describing a spray based on theory or measurement. Droplet size, droplet distribution, spray cone angle, and liquid break up length are among the important spray characteristics produced by an atomizer. These characteristics are influenced by various parameters including types of liquid, liquid physical properties, operating parameters, and atomizer geometries. Gas-to-liquid ratio (GLR) is an important operating parameter of twin-fluid atomizers due to the significant influence on resultant spray characteristics of twin-fluid atomizers. It is defined as:

$G L R=\frac{m_{G}}{m_{L}}$

Where $\dot{m}_{G}$ and $\dot{m}_{L}$ are mass flow rates of gas and liquid in $\mathrm{kg} / \mathrm{s}$, respectively.

Sovani et al. [1] suggested that the spray angle of an effervescent atomizer in a high pressure ambient conditions could be correlated as a function of GLR, injection pressure and ambient pressure. Jedelsky and Jicha [2] and Ochowiak et al. [3] observed increment of the spray angle in a swirl effervescent atomizer as GLR increased to maximum value of 0.07 . Increase of GLR has the same effect on the levels of turbulence of the jet discharging the atomizer as the increase of injection pressure. The increase of GLR corresponds to the increase of the velocity of injected gas thereby, creates a higher turbulence level of the discharging jet [1]. Ochowiak and Matuszak- [4] introduced gas bubbles to the existing atomizer to examine the effect of liquid aeration on the atomiza- tion process. The modification leads to decrease of the mean droplet diameter.

The flow structures inside an atomizer is also one of the factors determining the characteristics of spray produced. The two-phase flow is the most common internal flow structures exist inside an atomizer inclusive of the swirl effervescent atomizer. Two-phase flow regime inside a pipe differs with the orientation of the pipe. In one experimental study, Huang et al. [5] have classified flow in effervescent atomizer with vertical downward position into three regimes namely, bubbly flow, intermittent flow and annular flow. They also described that the flow transforms from bubbly to intermittent and finally annular with changes of GLR.

Some geometrical features that affect the resultant spray and internal flow structures of swirl effervescent atomizer are the swirl generators, mixing chamber diameter, aeration hole diameter, and discharge orifice diameter. Rho et al. [6] and Ghaffar et al. [7] constructed a twin-fluid atomizer with attached swirl-generating vane. They varied the swirl-generating vane angle and observed that a more intense swirling produced a wider spray angle. Zaremba et al. [8] compared four different atomizers and concluded that geometries were important parameters affecting spray formation especially at low GLR.

There are limited studies on swirl effervescent atomizer that discuss the critical parameters affecting the spray characteristics and internal flow structures. These parameters will further help in understanding the characteristics of swirl effervescent atomizer. Hence, effort has been made to find the most critical parameters that will produce the required spray characteristics and internal flow structure of the swirl effervescent atomizer. Results are presented as plots of three radar charts of the spray characteristics and internal flow structure (spray angle, breakup length, and gas core diameter) against swirl-generating vane, GLR, and discharge orifice diameter. 


\section{Methods}

The investigated swirl effervescent atomizer employs inside-out gas injection configuration that enables gas bubbles coming out from the aeration tube to mix with the bulk liquid in the mixing chamber. Liquid enters the mixing chamber of the atomizer through the side inlet and gas enters through the central inlet. The gas-liquid mixture experiences swirling phenomenon as passes through the swirl-generating vanes before exiting the atomizer through the discharge orifice. Perspex was used for the fabrication of the atomizer main body to facilitate internal flow visualization. The inner sides of the main body undergo surface finish process to ensure good transparency. This helps in visualizing the two-phase flow mixtures in the atomizer as it passes through the main body. The schematic of the investigated swirl effervescent atomizer is shown in Figure 1.

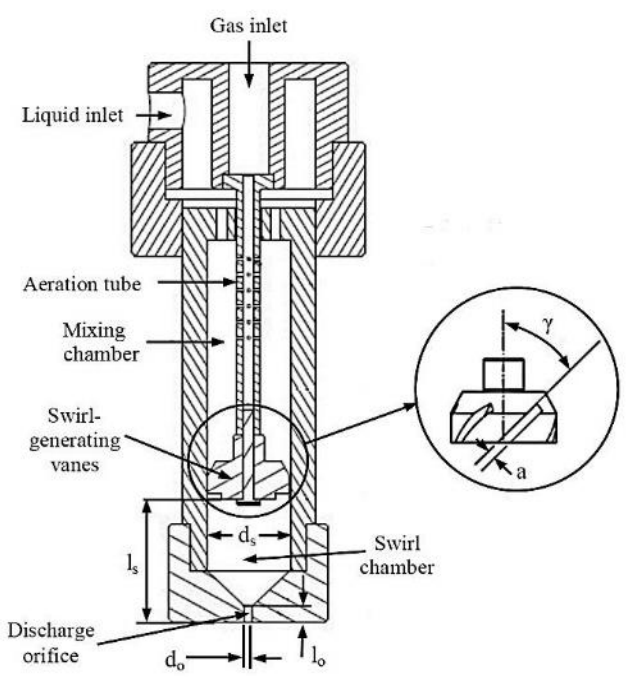

Fig. 1: Schematic of investigated swirl effervescent atomizer

An experimental test-rig was constructed to perform the performance test of the atomizer. The working fluid used was water while nitrogen acts as the atomization assistance. A line diagram of the experimental test-rig is shown in Figure 2. A pulseless centrifugal pump was utilized for delivering water from the water supply tank to the atomizer through the water-line. The amount of water flowing out of the pump was controlled by a ball valve installed at the outlet. Pressure regulator was used to regulate the amount of gas flow from the nitrogen gas cylinder to the atomizer. Measurement of water and gas flow rates in the system was obtained from water and gas flow transmitters respectively. The flow of both water and gas were controlled by globe valves. Water strainer was installed prior to the water flow transmitter inlet to prevent unwanted debris passing through the meter and led to malfunctioned [9]. Water and gas injection pressures were measured by digital pressure gauges. Water and gas flow check valves were installed at the inlet of the atomizer to allow unidirectional flow. The injector was fixed in vertical downward position and produce water sprays into a water collection tank. A submersible pump delivers the water back into the water supply tank to complete the cycle. The video recordings of the resultant sprays produced were captured by a high-speed camera with $800 \times 600$ video resolutions at 1000 frames per second. The shutter speed was set to a maximum value which results an exposure of $5 \mu \mathrm{s}$. Shadowgraph technique was applied in acquiring the resultant sprays video recordings. Data acquisition method of the spray angle, breakup length, and gas core diameter were explained in our previous work [10]-[12].

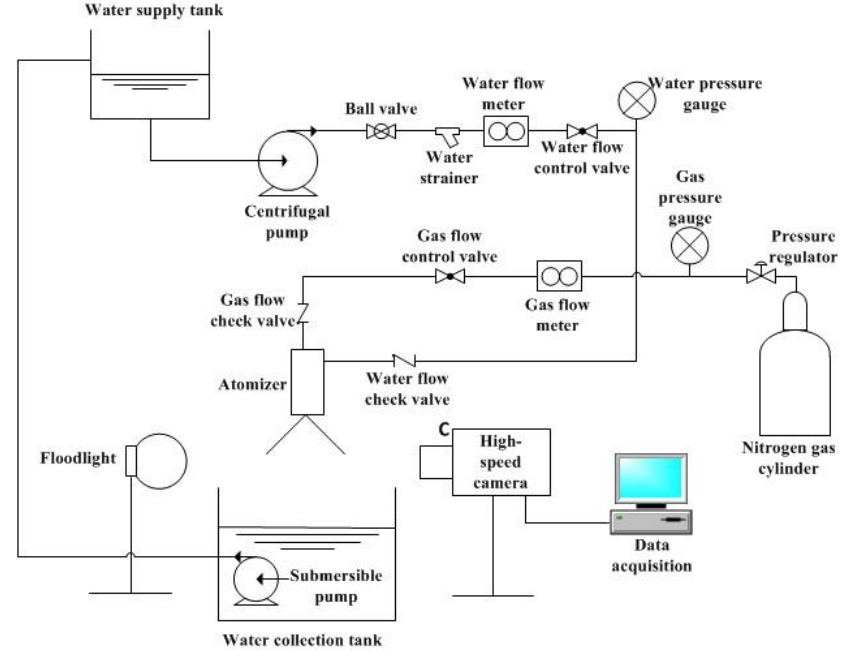

Fig. 2: Line diagram of test rig

The swirl-generating vane angle, GLR, and discharge orifice diameter were configured at three levels as shown in Table 1.

Table 1: Range of level for the independent parameters

\begin{tabular}{|l|c|c|c|}
\hline Level & $\begin{array}{c}\text { Swirl- } \\
\text { generating } \\
\text { vane angle }\left(^{\circ}\right.\end{array}$ & GLR & $\begin{array}{c}\text { Discharge } \\
\text { orifice } \\
\text { diameter (mm) }\end{array}$ \\
\hline Low & 30 & 0.0003 & 1.5 \\
\hline Medium & 45 & 0.0006 & 2.0 \\
\hline High & 60 & 0.0009 & 2.5 \\
\hline
\end{tabular}

\section{Results and Discussions}

The dependency level of spray angle to the swirl-generating vane angle, GLR, and discharge orifice diameter is illustrated in Figure 3.

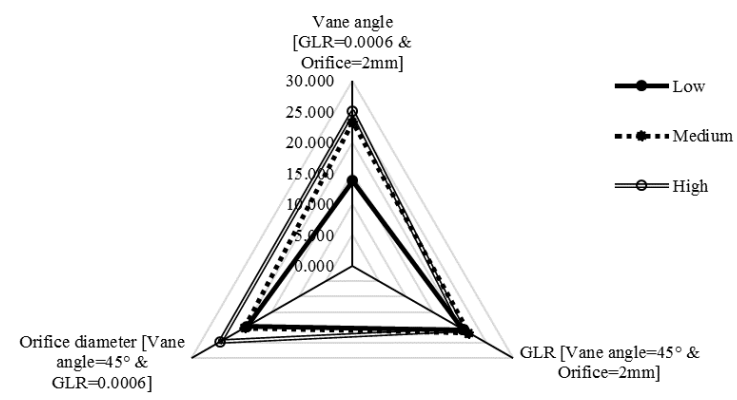

Fig. 3: Dependency level of spray angle to swirl-generating vane angle, GLR, and discharge orifice diameter

Among the three independent parameters, the widest spray angle $\left(\sim 25^{\circ}\right)$ is observed at the high level of swirl-generating vane angle and discharge orifice diameter. This occurrence is probably resulting from the association of swirl intensity of a liquid/liquid-gas mixture flowing in the atomizer with swirl-generating vane angle. The centrifugal pressure produced by the swirling liquid sheet converted to axial velocity component tends to widen the profile of the spray. A higher swirl intensity is associated with a higher centrifugal pressure and hence, widens the spray profile [13]. It is also shown that spray angle is narrower $\left(<25^{\circ}\right)$ at the high level of GLR. This is caused by the higher value of gas injection pressure in GLR compared to swirl-generating vane angle and discharge orifice as depicted in Table 2 to Table 4 . Increasing the gas injection pressure increases the dynamic force of atomizing gas forcing the formation of a narrow spray [14]. 
Table 2: Relation of swirl-generating vane angle with gas injection pressure

\begin{tabular}{|c|c|}
\hline Swirl-generating vane angle $\left(^{\circ}\right)$ & Gas injection pressure (bar) \\
\hline 30 & 1.064 \\
\hline 45 & 1.072 \\
\hline 60 & 1.151 \\
\hline
\end{tabular}

Table 3: Relation of GLR with gas injection pressure

\begin{tabular}{|c|c|}
\hline GLR & Gas injection pressure (bar) \\
\hline 0.0003 & 0.865 \\
\hline 0.0006 & 1.153 \\
\hline 0.0009 & 1.209 \\
\hline
\end{tabular}

Table 4: Relation of discharge orifice diameter with gas injection pressure \begin{tabular}{|l|l} 
Discharge orifice diameter $(\mathrm{mm})$ & Gas injection pressure (bar) \\
\hline
\end{tabular}

\begin{tabular}{|c|c|}
\hline Discharge orifice diameter $(\mathrm{mm})$ & Gas injection pressure (bar) \\
\hline 1.5 & 1.732 \\
\hline 2.0 & 1.158 \\
\hline 2.5 & 0.334 \\
\hline
\end{tabular}

Figure 4 shows the dependency level of breakup length to swirlgenerating vane angle, GLR, and discharge orifice diameter. The shortest breakup length was observed at the high level of swirlgenerating vane angle $(\sim 10 \mathrm{~mm})$. As discussed earlier, a high level of swirl-generating vane angle resulted the widest spray angle. A wider spray angle is associated with a shorter breakup length as found by Laryea and No [15]. This phenomenon is caused by the persistency of area of the liquid film. It is known that the area of the liquid film is dependent on the mass flowrate of the liquid. Since the liquid mass flowrate was held at a constant value, the area of the liquid film remained unchanged. With the widening of the liquid film of the spray profile, the length of the liquid film tends to become shorter to maintain the area of the liquid film. This resulted in the spray having a shorter breakup length.

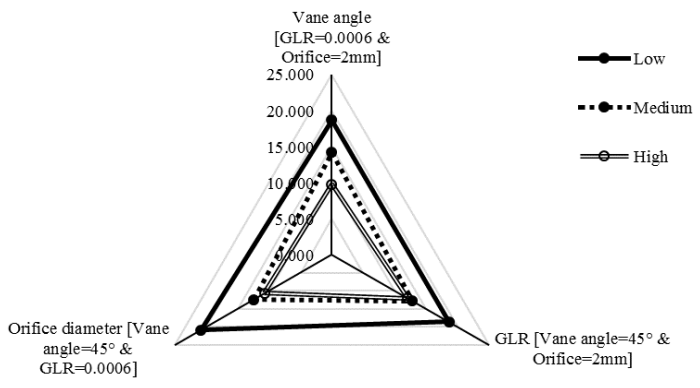

Fig. 4: Dependency level of breakup length to swirl-generating vane angle, GLR, and discharge orifice diameter

The high level of discharge orifice diameter has caused the breakup length to be only slightly longer than the shortest breakup length. This is resulted from the fact that effect of discharge orifice diameter on producing wide spray angle is lesser compared to the swirl-generating vane angle. High and medium levels of GLR have resulted in a breakup with almost the same length because there are only small differences of gas injection pressure between these two levels as shown in Table 3. Gas injection pressure is directly proportional to the bubble energy, i.e., energy to overcome the surface tension forces of the liquid jet. Hence, the small differences do not produce any significant changes in the spray breakup length.

Figure 5 depicts the dependency level of gas core diameter to swirl-generating vane angle, GLR, and discharge orifice diameter. It was observed that the largest gas core diameter occurred at the high level of GLR. This corresponds to the increase of the number and size of bubbles formed in the swirl chamber with the increase in GLR as shown in Figure 6. It is shown that the images of bubble formation inside the swirl chamber at different GLR. It is observed that as GLR increases, the size and number of bubbles also increases. Coalescence of bubbles with bigger size and number formed a gas core with larger diameter. At low GLR, the gas core diameter was observed to be the smallest compared to other parameters. The amount of GLR probably too low to form large bubbles that coalesce into a large gas core. This also shows that the formation of gas core is governed mostly on the amount of gas injected to the system.

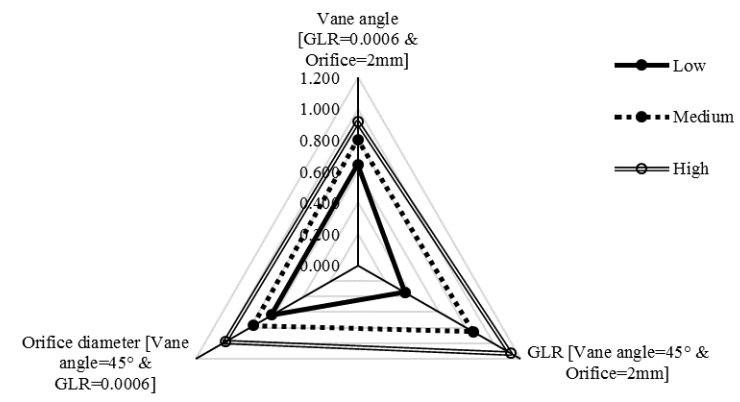

Fig. 5: Dependency level of gas core diameter to swirl-generating vane angle, GLR, and discharge orifice diameter

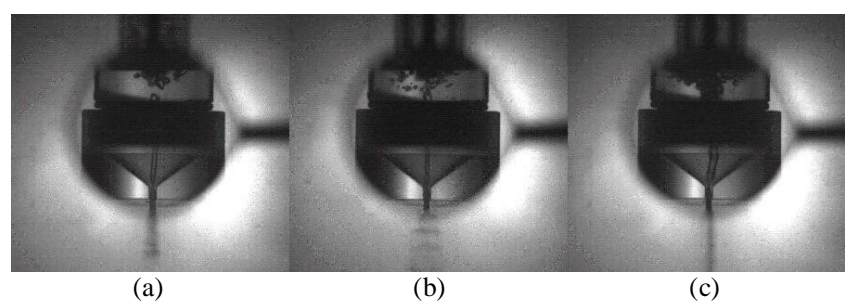

Fig. 6: Formation of bubbles at GLR (a) 0.0003 (b) 0.0006 and (c) 0.0009

\section{Conclusion}

The dependency level of the spray characteristics and internal flow structure of swirl effervescent atomizer to three independent parameters has been determined in the effort to find the most critical parameters affecting them. Geometrical parameters found to be more dominant for characterizing the resultant sprays, but operating conditions is more critical in affecting the internal flow structures. The widest spray angle $\left(\sim 25^{\circ}\right)$ and shortest breakup length $(\sim 10 \mathrm{~mm})$ were observed at the high level of swirl-generating vane angle and discharge orifice diameter. Gas core diameter expanded up to $1.13 \mathrm{~mm}$ at the high level of GLR. These results help in further understanding the characteristics of swirl effervescent atomizer.

\section{Acknowledgement}

The authors gratefully acknowledge the help of Universiti Teknologi MARA (UiTM) in providing the Geran Inisiatif Penyeliaan (GIP), Reference number: 600-IRMI/MyRA 5/3/GIP (009/2017) and the Research Management Center (RMC) of UiTM for facilitating this project.

\section{References}

[1] S. D. Sovani, E. Chou, P. E. Sojka, J. P. Gore, W. A. Eckerle, and J. D. Crofts, High pressure effervescent atomization: effect of ambient pressure on spray cone angle, Fuel 80:3 (2001) 427-435.

[2] J. Jedelsky and M. Jicha, Novel modifications of twin-fluid atomizers: performance, advantages and drawbacks, in 23th European Conference on Liquid Atomization and Spray Systems ILASS-Europe, Brno, Czech Republic, 6:8 (2010) 1-5.

[3] M. Ochowiak, L. Broniarz-Press, S. Rozanska, M. Matuszak, and S. Wlodarczak, Characteristics of spray angle for effervescent-swirl atomizers, Chemical Engineering Process: Process Intensification 98 (2015) 52-59.

[4] M. Ochowiak and M. Matuszak, The effect of additional aeration of liquid on the atomization process for a pneumatic nebulizer, European Journal of Pharmaceutical Sciences 97 (2017) 99-105.

[5] X. Huang, X. Wang, and G. Liao, Visualization of two phase flow inside an effervescent atomizer, Journal of Visualization 11:4 (2008) 299-308.

[6] B. J. Rho, S. J. Kang, J. H. Oh, and S. G. Lee, Swirl effect on the spray characteristics of a twin-fluid jet, KSME International Journal, 
12:5 (1998) 899-906.

[7] Z. A. Ghaffar, S. Kasolang, A. H. A. Hamid, M. A. A. Bakar, and C. S. Ow, Effect of Geometrical Parameters Interaction on Swirl Effervescent Atomizer Spray Angle, Jurnal Teknologi 76:9 (2015) 63-67.

[8] M. Zaremba, L. Weiß, M. Malý, M. Wensing, J. Jedelský, and M. Jícha, Low-pressure twin-fluid atomization: Effect of mixing process on spray formation, International Journal of Multiphase Flow 89 (2017) 277-289.

[9] Hedland, MR Flow Transmitter Installations and Programming Instructions, HLIT 306 (2008).

[10] Z. A. Ghaffar, S. Kasolang, and A. H. A. Hamid, Characteristics of swirl effervescent atomizer spray angle, 607 (2014).

[11] Z. A. Ghaffar, S. Kasolang, A. H. A. Hamid, C. S. Ow, and N. R. Nik Roselina, Design, development and performance evaluation of new swirl effervescent injector, Jurnal Teknologi, 75:1 (2015).

[12] Z. A. Ghaffar, S. Kasolang, A. H. A. Hamid, A. Diyar I., K. I. Sainan, and N. R. Nik Roselina, Gas Core Characteristics of Swirl Effervescent Atomizer, Jurnal Teknologi, 76:9 (2015) 57-62.

[13] V. Yang, M. Habiballah, J. Hulka, and M. Popp, Liquid rocket thrust chambers: aspects of modeling, analysis, and design, Progress in Astronautics and Aeronautics, 2004.

[14] L. Juslin, O. Antikainen, P. Merkku, and J. Yliruusi, Droplet size measurement: I. Effect of three independent variables on droplet size distribution and spray angle from a pneumatic nozzle, International Journal of Pharmaceutics 123:2 (1995) 247-256.

[15] G. N. Laryea and S. Y. No, Spray angle and breakup length of charge-injected electrostatic pressure-swirl nozzle, Journal of Electrostatics, 60:1 (2004) 37-47. 